\title{
Reseña de sistemas de control holónicos para viviendas
}

\section{Review of holonic control systems for dwelling}

Presentación: 06/10/2020

\section{Doctorando:}

\section{Germán Rodolfo Henderson}

Grupo CLIOPE, Facultad Regional Mendoza, Universidad Tecnológica Nacional - Argentina german.henderson@docentes.frm.utn.edu.ar

\section{Director:}

\section{Alejandro Pablo Arena}

\section{Co-director:}

\section{Facundo Bromberg}

\section{Resumen}

El sector residencial representa un elevado porcentaje del consumo de energía global y por ello nacen muchos sistemas de domótica. En el año 1968 surgen los sistemas holónicos, con un gran crecimiento en las últimas décadas. Sin embargo, no existe una revisión de esta clase de sistemas aplicados al sector residencial. Se presenta aquí una primera revisión en este campo, en la que se plantean los desafíos que hay por delante.

Palabras clave: holón, holarquía, control, vivienda.

\begin{abstract}
The residential sector represents a high percentage of global energy consumption and therefore many home automation systems are developed. In 1968, holonic systems emerged, with considerable growth in recent decades. However, there is no review on such systems being applied to the residential sector. A first revision is presented here in this field, which raises the challenges ahead.
\end{abstract}

Keywords: holon, holarchy, control, dwelling.

\section{Introducción}

A nivel mundial, el consumo de energía final en el sector residencial representa aproximadamente el 40\% del total (B. P.1.c., 2019). Con el objetivo de hacer un uso más eficiente de la energía y con la reducción del coste y el avance de las tecnologías de la información y la comunicación, los sistemas de gestión de edificios (BMS) se han desarrollado durante las dos últimas décadas (Singh et al., 2020).

Se han propuesto muchas soluciones, con un gran predominio de la lógica difusa en los sistemas de control por su gran adaptabilidad a los mismos (Singh et al., 2020). Sin embargo, existen algunas limitaciones en los casos en que los problemas a resolver son heurísticos (Singh et al., 2020), lo cual es común cuando se considera la comodidad de los usuarios en un hogar.

En 1968, Arthur Koestler introdujo el término holón, que sugiere una perspectiva compleja, considerando los sistemas de manera holística y reduccionista al mismo tiempo (Koestler, 1967). Un holón es un elemento completo que puede tomar decisiones por sí mismo, pero es parte de algo más grande, un holón de jerarquía superior. Además, puede estar formado por unidades más pequeñas, holones de jerarquías inferiores. 
A partir de esto, Koestler definió el término holarquía, que significa una jerarquía de holones (Koestler, 1967). Los holones tienen extremos abiertos tanto en dirección ascendente como descendente, por lo que siempre puede encontrar elementos más pequeños o elementos más grandes que lo envuelvan.

El objetivo de este trabajo fue revisar la literatura sobre los sistemas holónicos aplicados a la vivienda, determinando los desafíos actuales en este campo.

\section{Desarrollo}

\section{Revisión de la literatura}

Para la búsqueda de artículos se utilizaron las herramientas Scopus and Google Scholar (GS) ingresando las palabras clave dwelling AND control. Los resultados fueron 8.354 y 15.200 publicaciones, respectivamente. Una búsqueda más afinada con las palabras clave (holonic AND control) OR ("holonic system") AND (house OR dwelling OR home OR building), redujeron los resultados a 39 y 29 publicaciones, respectivamente en Scopus y GS. En la Figura 1(a) se pueden ver el número de publicaciones a lo largo de los años.

Revisando cada uno de ellos, se identificaron 7 Sistemas de Control Holónico (SCH) aplicados en edificaciones y ninguno específicamente a vivienda. Debido al pequeño número de publicaciones encontradas, se amplió la investigación. Por lo tanto, se consideraron Otros Sistemas de Control (OSC) para viviendas.

En general, la distribución de los artículos encontrados se muestra en la Figura 1(b). Es claro que la mayor parte de las investigaciones realizadas es en el ámbito de la ciencias de la computación y la ingeniería, integrando entre ambas el 52.4\%. Sin embargo, es necesario potenciar las investigaciones en temas más específicos como el de la Energía, que al momento solo abarca un 1.3\% del total de las investigaciones.

\section{A. Sistemas de Control Holónicos para Viviendas}

Entre las características de los sistemas holónicos, podemos definir como principales a:

- su funcionamiento bajo reglas fijas y estrategias variables a partir del autoaprendizaje por retroalimentación, que le brindan al sistema flexibilidad.

- Escalabilidad dinámica, ajustándose a la falta de partes o presencia de nuevas.

- Bisociación y disociación, que le permite tener adaptabilidad para componer o disociar jerarquías más grandes o más chicas a partir del conocimiento de cada una de ellas, respectivamente.

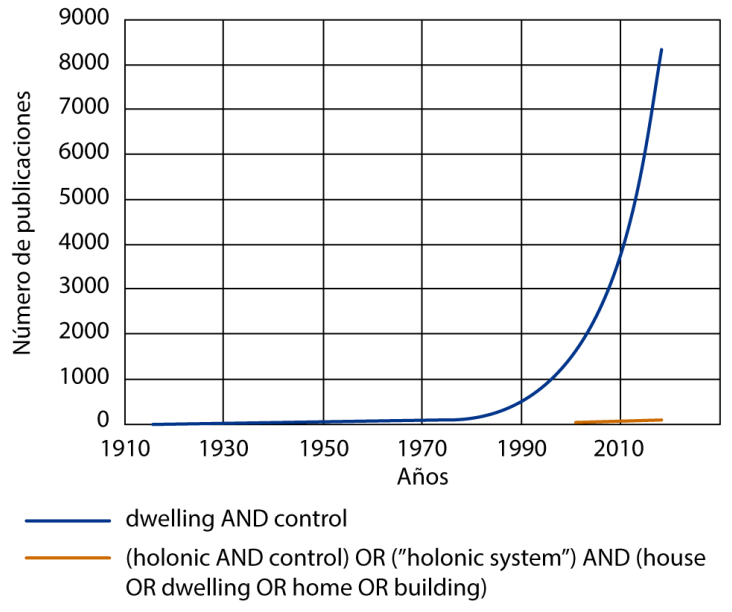

(a)

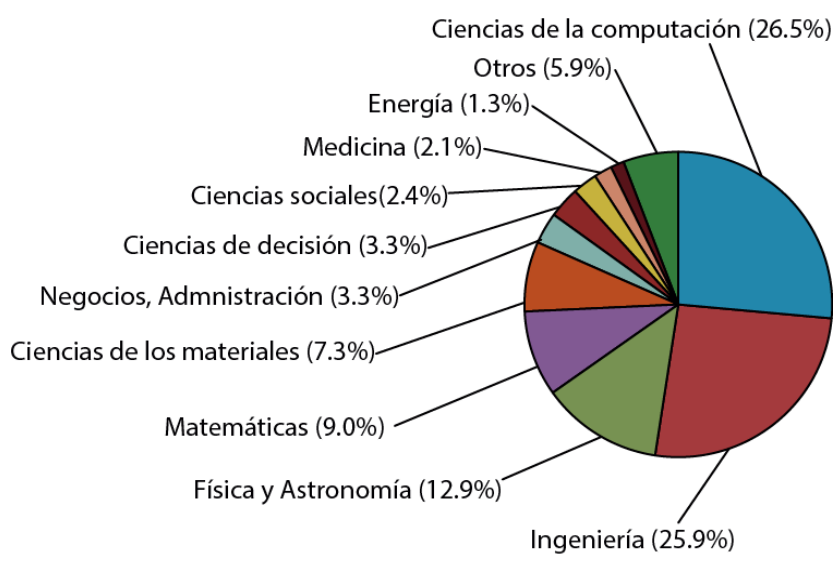

Copyright $\odot 2019$ Elsevier B.V.Todos los derechos reservados. Scopus ${ }^{\bullet}$ es una marca registrada de Elsevier B.V.

(b)

Figura 1: (a) Diagrama Agente-Entorno a utilizar en el trabajo y (b) Distribución en áreas temáticas de las publicaciones. 
Todas estas características junto con otras permiten que la vivienda evolucione gradualmente, ajustándose a la capacidad de los habitantes para alcanzar la tecnología y al sistema para adaptarse a nuevas aplicaciones.

\section{B. Otros Sistemas de Control para Viviendas}

Existen otros tipos de sistemas que se han desarrollado para automatizar viviendas o edificios. Todos ellos pueden integrarse en los sistemas holónicos sin grandes cambios. Por tanto, es importante tenerlos en cuenta.

Los sistemas multiagente son los más parecidos a los holónicos cuando se analizan sus características, por lo que son los que mejor pueden adaptarse. Es posible decir que un holón es un tipo particular de agente (Van Belle et al., 2009). Por este motivo, son estos los elegidos para profundizar la revisión de literatura.

\section{Análisis}

Para un análisis de los sistemas de control revisados, se caracterizan según el servicio a automatizar, tomando como referencia los considerados por (Sauter et al., 2011), que son: Climatización (CC), Confort Visual (VC), Seguridad (SA) (Safety), Seguridad (SE) (Security), Transporte, Audio unidireccional, Gestión de energía (EM), Suministro y Disposición final, Comunicación e intercambio de información (C\&Ie) y Varios dominios especiales. Además, se han incorporado la comodidad del usuario (UC) y la calidad del aire (AQ), como se considera en varios de los trabajos revisados. El servicio genérico se refiere a cuando los autores no especifican uno y cualquiera puede postularse.

Además, se consideraron los tipos de edificios a los que se han aplicado y el sistema de control utilizado. De la misma forma que en servicios, tipo genérico de edificación se refiere a cuando los autores no especifican uno y cualquiera podría postular. El número de elementos considerados de esta manera fue $20 \mathrm{y}$ se muestran en la Tabla I.

Se revisaron diversos aspectos de cada sistema, encontrando características que permiten identificar brechas en los sistemas.

Primero, para el SCH, no se ha encontrado una definición clara de los sistemas que lo caracterizan como tal. En otras áreas de mayor madurez, como los procesos de fabricación, ya se han logrado avances (Leitão et al., 2003). También se encontró como factor común que no se especifican los límites de la SCH. Esto se considera esencial para la interoperabilidad y la compresión de sistemas.

En segundo lugar, aquellos estudios que contemplan los servicios CC o EM lo han hecho para lograr ahorros energéticos. En algunos estudios se ha considerado la UC, que ha demostrado ser más compleja. Los resultados son prometedores a este respecto.

Finalmente, una de las potencialidades de esta clase de sistemas es la interoperabilidad que sugieren con otros niveles de jerarquía. El holón Vivienda participa en muchos sistemas como energía, agua, gas, transporte y otras redes de distribución. (Howell et al., 2017) sugieren el uso de sistemas holónicos para el futuro de las redes inteligentes con recurso distribuido, considerando el almacenamiento distribuido y la generación de energía.

Se puede decir que el holón Vivienda será el corazón de los futuros sistemas que conformarán las grandes ciudades. Pero es necesario lograr una madurez en los sistemas de holarquías inferiores, lo que estará motivado por el crecimiento de los holones de holarquías superiores.

Otros aspectos que han sido relevantes en los estudios revisados son los de AQ, SA y SE, campos en los que aún queda mucho trabajo por hacer.

\section{Resultados}

\section{Desafíos}

El desafío más importante que se ha encontrado es la correcta definición y comprensión de estos sistemas. Es necesario desarrollar estándares para la definición de esta clase de sistemas.

Por otro lado, es fundamental experimentar más con este tipo de sistemas en todos los niveles de jerarquía. 
El desarrollo de indicadores para la optimización de los SCH ha tenido sus avances en el campo de la fabricación, que es el campo más desarrollado en cuanto a estos sistemas, pero es necesario experimentarlos en otros campos y desarrollar otros nuevos que permitan la mejora continua.

Otro desafío asociado al pobre desarrollo de una ontología es la pérdida de flexibilidad y confiabilidad en las holarquías. Cuando un holón cambia, puede afectar a otros creando grandes problemas (Mathews, 1996).

\section{Conclusiones y discusión}

Aquí se presentó una primera revisión de la literatura sobre sistemas de control holónico para viviendas. Se concluye que hasta el momento se han realizado pocas investigaciones.

Es necesario definir una ontología para estos sistemas como lo han hecho (Bonino and Corno, 2008) para los entornos inteligentes para permitir la interoperabilidad. Es importante identificar los límites de los holones para que permitan una expansión y reducción de la holarquía.

\begin{tabular}{|c|c|c|c|}
\hline Referencia & Servicio & Tipo de edificio & Sistema de Control \\
\hline (Brooks et al., 1997) & ac\&le & Cuarto de laboratorio & Multiagentes \\
\hline (Boman et al., 1998) & EM, UC & Comercial & Multiagentes \\
\hline $\begin{array}{c}\text { (Sharples et al., } \\
1999)\end{array}$ & $\mathrm{EM}, \mathrm{UC}, \mathrm{SA}$ & Residencial & Multiagentes \\
\hline (Fu and Shih, 2000) & Genérico & Genérico & Sistema Holónico \\
\hline $\begin{array}{l}\text { (Luo, Lin, and Su, } \\
\text { 2003) }\end{array}$ & SA, SE & Genérico & Multiagentes \\
\hline (Hagras et al., 2000) & SA, UC & Genérico & Multiagentes \\
\hline (Hagras et al., 2003) & SA, UC & Genérico & $\begin{array}{c}\text { Aprendizaje Reforzado y } \\
\text { Multiagentes }\end{array}$ \\
\hline $\begin{array}{l}\text { Davidsson and } \\
\text { Boman, 2005) }\end{array}$ & EM, CC & Genérico & Multiagentes \\
\hline $\begin{array}{l}\text { (Rutishauser, Joller, } \\
\text { and Douglas, 2005) }\end{array}$ & EM, UC, SA & Comercial & Multiagentes \\
\hline $\begin{array}{l}\text { (Duan and Lin, } \\
\text { 2008) }\end{array}$ & CC, VC & Comercial & Multiagentes \\
\hline (Bonino et al., 2009) & C\&le & Residencial & Reglas fijas en Java \\
\hline $\begin{array}{l}\text { (Bonino and Corno, } \\
2010 \text { ) }\end{array}$ & C\&le & Residencial & API Jena \\
\hline $\begin{array}{c}\text { (Bonino and Corno, } \\
\text { 2011) }\end{array}$ & C\&le & Residencial & API Jena \\
\hline (Silva et al., 2012) & Generic & Genérico & Sistema Holónico \\
\hline $\begin{array}{c}\text { (Santamaria et al., } \\
\text { 2014) }\end{array}$ & EM, UC & Residencial & $\begin{array}{c}\text { Arquitectura de Servicios } \\
\text { Orientados }\end{array}$ \\
\hline $\begin{array}{l}\text { (Galland and Gaud, } \\
\text { 2015) }\end{array}$ & In & Genérico & Sistema Holónico \\
\hline $\begin{array}{l}\text { (Carbonari et al., } \\
\text { 2018) }\end{array}$ & EM, UC & Comercial & Sistema Holónico \\
\hline (Pirani et al., 2018) & EM, UC & Comercial & Sistema Holónico \\
\hline $\begin{array}{c}\text { (Carbonari et al., } \\
\text { 2019) }\end{array}$ & $\mathrm{CC}, \mathrm{AQ}$ & Comercial & Sistema Holónico \\
\hline $\begin{array}{c}\text { (Naticchia et al., } \\
\text { 2019) } \\
\end{array}$ & EM, SA & Genérico & Sistema Holónico \\
\hline
\end{tabular}

Tabla 1: Caracterización de la literatura revisada 
Estos sistemas presentan en los estudios una potencial reducción del consumo energético que podría afectar significativamente la demanda energética del sector residencial y sus consiguientes emisiones de gases contaminantes. Además, existen interesantes aplicaciones de $\mathrm{SCH}$, como las posibilidades de análisis de refuncionalización de edificios considerando el consumo energético (Carbonari et al., 2018).

Para mejorar el uso de los sistemas de control en el sector residencial, se deben desarrollar equipos de bajo costo y fáciles de usar. La comodidad de los usuarios debe tenerse en cuenta en todos los desarrollos porque una mejora real en la comodidad de los usuarios hará que el uso de los sistemas sea exitoso. En consecuencia, la inteligencia artificial tiene un futuro prometedor.

Finalmente, es importante aprovechar los desarrollos existentes en la automatización de edificios, modificándolos sutilmente para que puedan adaptarse a sistemas con una perspectiva holónica y así aportar sus ventajas.

\section{Referencias}

Boman, M.; Davidsson, P.; Skarmeas, N.; Clark, K.; and Gustavsson, R. (1998). Energy Saving and Added Customer Value in Intelligent Buildings. Paam, vol. 1, pp. 505-516, doi: 10.1.1.41.6239.

Bonino, D.; Castellina, E.; Corno, F.; and Liu, M. (2009). Technology independent interoperation of domotic devices through rules. 2009 IEEE 13th International Symposium on Consumer Electronics, pp. 971-975, doi: 10.1109/ISCE.2009.5157026.

Bonino, D.; and Corno, F. (2008). DogOnt - Ontology modeling for intelligent domotic environments. Lecture Notes in Computer Science (including subseries Lecture Notes in Artificial Intelligence and Lecture Notes in Bioinformatics), vol. 5318 LNCS, pp. 790-803, doi: 10.1007/978-3-540-88564-1-51.

Bonino, D. and Corno, F. (2010). Rule-based intelligence for domotic environments. Autom. Constr., vol. 19, no. 2, pp. 183-196, doi: 10.1016/j.autcon.2009.10.008.

Bonino, D. and Corno, F. (2011). Modeling, simulation and emulation of Intelligent Domotic Environments. Autom. Constr., vol. 20, no. 7, pp. 967-981, doi: 10.1016/j.autcon.2011.03.014

B. P.1.c. (2019). BP Energy Outlook 2019 Edition.

Brooks, R. A. et al. (1997). The Intelligent Room Project. Proc. Second Int. Conf. Cogn. Technol., pp. 271-278, Accessed: May 06, 2019. [Online]. Available:

http://citeseerx.ist.psu.edu/viewdoc/download?doi=10.1.1.137.1674\&rep=rep1\&type=pdf.

Carbonari, A.; Messi, L.; Naticchia, B.; Vaccarini, M.; and Pirani, M. (2018). Development of a BIM-based cyber-physical system for facility management of buildings. Creative Construction Conference 2018 -

Proceedings, pp. 1028-1035, doi: 10.3311/CCC2018-133.

Carbonari, A.; Messi, L.; Naticchia, B.; Vaccarini, M.; and Pirani, M. (2019). Development of a BIM-based holonic system for real-time monitoring of building operational efficiency. Front. Eng. Manag., pp. 1-15, doi: 10.1007/s42524-019-0037-0.

Davidsson, P. and Boman, M. (2005). Distributed monitoring and control of office buildings by embedded agents. Inf. Sci. (Ny)., vol. 171, no. 4, pp. 293-307, doi: 10.1016/j.ins.2004.09.007.

Duan, J.; and Lin, F. (2008). Research of intelligent building control using an agent-based approach. IEEE Int. Conf. Ind. Informatics, pp. 991-994, 2008, doi: 10.1109/INDIN.2008.4618246.

Fu, L. C.; and Shih, T. J. (2000). Holonic supervisory control and data acquisition kernel for 21st century intelligent building system. Proceedings - IEEE International Conference on Robotics and Automation, vol. 3, pp. 2641-2646, doi: 10.1109/robot.2000.846426.

Galland, S. and Gaud, N. (2015). Organizational and holonic modelling of a simulated and synthetic spatial environment. Lecture Notes in Computer Science (including subseries Lecture Notes in Artificial Intelligence and Lecture Notes in Bioinformatics), vol. 9068, pp. 147-169, doi: 10.1007/978-3-319-23850-0_10. 
Hagras, H.; Callaghan, V.; Colley, M.; and Clarke, G. (2000). A Hierarchical Fuzzy Genetic Multi-Agent Architecture for Intelligent Buildings Sensing and Control. Developments in Soft Computing, pp. 199-206.

Hagras, H.; Callaghan, V.; Colley, M.; and Clarke, G. (2003). A hierarchical fuzzy-genetic multi-agent architecture for intelligent buildings online learning, adaptation and control. Information Sciences, vol. 150, no. 1-2, pp. 33-57, doi: 10.1016/S0020-0255(02)00368-7.

Howell, S.; Rezgui, Y.; Hippolyte, J. L.; Jayan, B.; and Li, H. (2017). Towards the next generation of smart grids: Semantic and holonic multi-agent management of distributed energy resources. Renewable and Sustainable Energy Reviews, vol. 77. pp. 193-214, doi: 10.1016/j.rser.2017.03.107.

Koestler, A. (1967). The ghost in the machine, First edit. New York, United Stated: The Macmillan Company. Leitão, P.; Colombo, A. W.; Restivo, F.; and Schoop, R. (2003). Formal specification of holonic control system ADACOR product holon, using high-level Petri Nets. IEEE Int. Conf. Ind. Informatics, vol. 2003-Janua, pp. 263-272, doi: 10.1109/INDIN.2003.1300278.

Luo, R. C.; Lin, S. Y.; and Su, K. L. (2003). A multiagent multisensor based security system for intelligent building. IEEE Int. Conf. Multisens. Fusion Integr. Intell. Syst., pp. 311-316, 2003, doi: 10.1109/MFI-2003.2003.1232676.

Mathews, J. (1996). Holonic organisational architectures. Hum. Syst. Manag., vol. 15, no. 1, pp. 27-54, doi: 10.3233/HSM-1996-15105.

Naticchia, B.; Messi, L.; Pirani, M.; Bonci, A.; Carbonari, A.; and Tolve, L. C. (2019). Holonic system for real-time emergency management in buildings. Proc. 36th Int. Symp. Autom. Robot. Constr. ISARC 2019, no. Isarc, pp. 453-460, doi: 10.22260/isarc2019/0061.

Pirani, M.; Messi, L.; Carbonari, A.; Bonci, A.; and Vaccarini, M. (2018). Holonic management systems for resilient operation of buildings,. ISARC 2018 - 35th International Symposium on Automation and Robotics in Construction and International AEC/FM Hackathon: The Future of Building Things, doi: 10.22260/isarc2018/0059.

Rutishauser, U.; Joller, J.; and Douglas, R. (2005). Control and learning of ambience by an intelligent building. IEEE Trans. Syst. Man, Cybern. Part ASystems Humans., vol. 35, no. 1, pp. 121-132, 2005, doi: 10.1109/TSMCA.2004.838459.

Santamaria, A. F.; De Rango, F.; Falbo, D.; and Barletta, D. (2014). SmartHome: a domotic framework based on smart sensing and actuator network to reduce energy wastes. Wireless Sensing, Localization, and Processing IX, vol. 9103, p. 910308, doi: 10.1117/12.2053328.

Sauter, T.; Soucek, S.; Kastner, W.; and Dietrich, D. (2011). The evolution of factory and building automation. IEEE Ind. Electron. Mag., vol. 5, no. 3, pp. 35-48, doi: 10.1109/MIE.2011.942175.

Sharples, S.; Callaghan, V.; and Clarke, G. (1999). Multi-agent architecture for intelligent building sensing and control. Sens. Rev., vol. 19, no. 2, pp. 135-140, doi: 10.1108/02602289910266278.

Silva, R. M.; Arakaki, J.; Junqueira, F.; Santos Filho, D. J.; and Miyagi, P. E. (2012). Modeling of active holonic control systems for intelligent buildings. Autom. Constr., vol. 25, pp. 20-33, doi: 10.1016/j.autcon.2012.04.002.

Singh, A. K.; Agrawal, S.; Agarwal, S.; and Goyal, D. (2020). Low-Cost and Energy-Efficient Smart Home Security and Automation. Computational Network Application Tools for Performance Management, M. Pant, T. K. Sharma, S. Basterrech, and C. Banerjee, Eds. Singapur, pp. 95-108.

Van Belle, J.; Saint Germain, B.; Verstraete, P.; Valckenaers, P.; Ali, O.; Van Brussel, H.; and Cattrysse, D. (2009). A holonic chain conveyor control system: An application. International Conference on Industrial Applications of Holonic and Multi-Agent Systems, Springer, Berlin, Heidelberg, pp. 234-243. 